Received: 13 March 2017

Accepted: 2 December 2017

Published online: 08 January 2018

NTIFIC REP RTS

\title{
High content image analysis reveals function of miR-124 upstream of Vimentin in regulating motor neuron mitochondria
}

\begin{abstract}
TalYardeni ${ }^{1,5}$, Raquel Fine ${ }^{1}$, Yuvraj Joshi ${ }^{2}$, Tal Gradus-Pery ${ }^{2}$, Noga Kozer ${ }^{3}$, Irit Reichenstein ${ }^{1}$, Eran Yanowski ${ }^{1}$, Shir Nevo ${ }^{1}$, Hila Weiss-Tishler ${ }^{1}$, Michal Eisenberg-Bord ${ }^{1}$, Tal Shalit ${ }^{4}$, Alexander Plotnikov ${ }^{3}$, Haim M. Barr ${ }^{3}$, Eran Perlson ${ }^{2}$ \& Eran Hornstein ${ }^{1}$

microRNAs (miRNAs) are critical for neuronal function and their dysregulation is repeatedly observed in neurodegenerative diseases. Here, we implemented high content image analysis for investigating the impact of several miRNAs in mouse primary motor neurons. This survey directed our attention to the neuron-specific miR-124, which controls axonal morphology. By performing next generation sequencing analysis and molecular studies, we characterized novel roles for miR-124 in control of mitochondria localization and function. We further demonstrated that the intermediate filament Vimentin is a key target of miR-124 in this system. Our data establishes a new pathway for control of mitochondria function in motor neurons, revealing the value of a neuron-specific miRNA gene as a mechanism for the re-shaping of otherwise ubiquitously-expressed intermediate filament network, upstream of mitochondria activity and cellular metabolism.
\end{abstract}

microRNAs (miRNAs) are endogenous non-coding RNAs that facilitate sequence-dependent posttranscriptional silencing, playing pivotal roles in brain development and neuronal function ${ }^{1-3}$. miRNA activity is required for motor neuron survival ${ }^{4}$ and broad dysregulation of miRNA biogenesis is associated with Amyotrophic Lateral Sclerosis (ALS) ${ }^{4-8}$. Several miRNA genes have already been suggested to play critical roles in motor neurons,

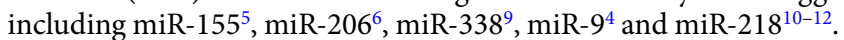

Mitochondria are cytoplasmic organelles implicated in ATP synthesis, calcium ion homeostasis and apoptotic cascades. Mitochondria are abundant in neurons, because of their high energetic demands ${ }^{13}$. Accordingly, mitochondria regulate axonal growth ${ }^{14,15}$. Mitochondrial dysfunction is implicated in neurodegeneration, including in Alzheimer's Disease $(\mathrm{AD})^{16}$, Parkinson's Disease $(\mathrm{PD})^{17}$, Huntington's Disease $(\mathrm{HD})^{8}$ and $\mathrm{ALS}^{18}$. For example, in ALS and in PD, impaired axonal transport causes abnormal accumulation of mitochondria in proximal axons $s^{17,19}$.

Functional interconnections between miRNAs and mitochondria were suggested by the existence of mature and precursor miRNAs in purified mitochondria ${ }^{20-22}$ and by the involvement of mitochondrial activity in RNA induced silencing complex (RISC) assembly and miRNA-mediated silencing ${ }^{23,24}$. Furthermore, miRNAs control nuclear-encoded mitochondrial protein $\mathrm{s}^{25}$ and mitochondrial metabolism ${ }^{26,27}$. Intriguingly, in neurons miRNAs regulate mitochondrial electron $\operatorname{transfer}^{28}$, respiration ${ }^{29}$ and pro-apoptotic mitochondrial cytochrome $\mathrm{c}$ release ${ }^{30}$.

In the current report we implemented high content image analysis for investigating the impact of miRNAs on primary motor neurons. We demonstrate that miR-124 overexpression impacts motor neuron morphology and mitochondrial activity. By performing next generation sequencing and molecular studies, we identified the intermediate filament Vimentin (Vim) as an important target of miR-124 in this new pathway. Vim is known to physically associate with mitochondria in different cell types, controlling its position and metabolic activity ${ }^{31,32}$. We show that a new miR-124-Vim pathway regulates mitochondria function and localization, at least in part via

\footnotetext{
${ }^{1}$ Department of Molecular Genetics, Weizmann Institute of Science, Rehovot, 7610001, Israel. ${ }^{2}$ Department of Physiology and Pharmacology, Sackler Faculty of Medicine, Tel Aviv University, Tel Aviv, Israel. ${ }^{3} \mathrm{HTS}$ unit, G-INCPM, Weizmann Institute of Science, Rehovot, 7610001, Israel. "'Bioinformatics unit, G-INCPM, Weizmann Institute of Science, Rehovot, 7610001, Israel. ${ }^{5}$ Present address: Center for Mitochondrial and Epigenomic Medicine, Children's Hospital of Philadelphia, Philadelphia, Pennsylvania, USA. TalYardeni and Raquel Fine contributed equally to this work. Correspondence and requests for materials should be addressed to E.H. (email: eran.hornstein@weizmann.ac.il)
} 
a

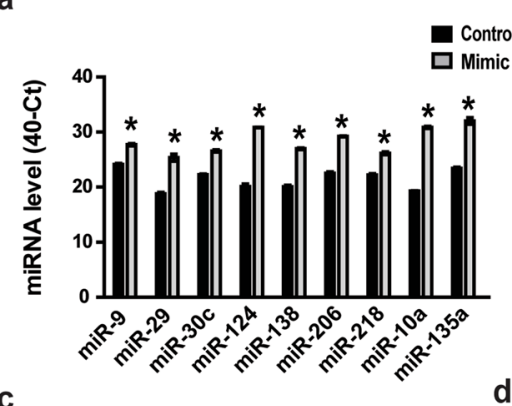

b

$\begin{array}{ccc}\begin{array}{c}\text { Spinal motor neuron } \\ \text { cultured on }\end{array} & \begin{array}{c}\text { Automated miRNA } \\ \text { mimic transfection }\end{array} & \begin{array}{c}\text { Automated fluorescence } \\ \text { microscopy \& morphometry }\end{array}\end{array}$

d

384 - well microplates
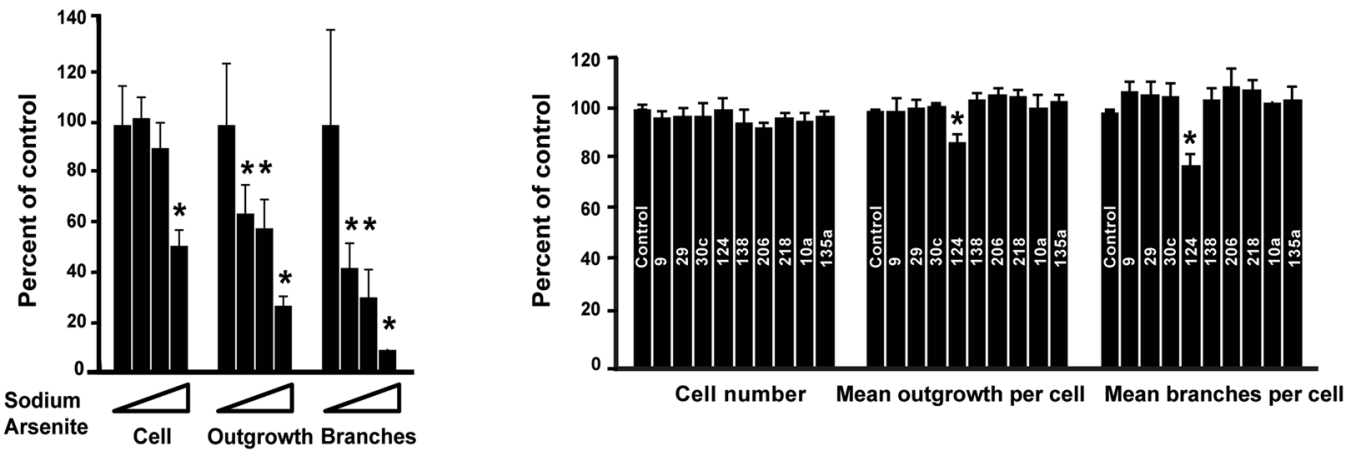

Figure 1. High content image analysis reveals the impact of miR-124 on primary motor neuron morphology. (a) Values for nine individual miRNAs, all transfected at $0.5 \mathrm{ng} / \mu \mathrm{l}$, to mouse primary motor neurons. miRNA expression levels displayed on the Y-axis as 40 minus qPCR cycle threshold (40-Ct), on a $\log _{2}$ scale. All miRNAs were significantly overexpressed. (b) A diagram describing the method: Spinal motor neurons were isolated from E13.5 mouse embryos and seeded on a 384 multiwell plate. Culture was transfected with different miRNA mimics using Bravo automated liquid handling robot. $72 \mathrm{hrs}$ later, cells were fixed, stained with anti Tuj1 antibody and DAPI. Two fluorescent micrographs were captured per well (ImageXpress Micro and MetaXpress2 software, Molecular Devices). (c) Cell numbers (Cell), neurite outgrowth per cell (outgrowth) and number of branches per cell (branches), were quantified with serial doses of the stress-inducing agent, Sodium Arsenite $(15,30$ and $60 \mu \mathrm{M}$, for 60 minutes). See Methods and Sup. Figure 2. (d) None of the nine miRNAs tested influenced cell numbers. miR-124 was the only miRNA to reduce mean axonal outgrowth per cell and mean number of branches per cell. 500 Tuj1+ neurons quantified per field, 2 fields/well and 6 wells per treatment in five independent experimental repeats. Data collected from $>30,000$ Tuj $1+$ neurons per treatment. Averages \pm SEM, Student's t-test. *P-value $<0.05$.

control of axonal transport. Our study reveals that Vim functions as a regulator of mitochondrial activity in motor neurons, downstream of miR-124.

\section{Results}

Here, we tested the impact of miRNAs on motor neuron morphology and function, which led us to discover a new pathway for regulation of mitochondria activity, downstream of miR-124.

We first calibrated a transfection system for miRNA in primary mouse motor neurons ${ }^{33}$. We isolated motor neurons from embryonic spinal cords, following ${ }^{34}$, and transfected a hematopoietic miRNA, miR-142, or a scrambled dsRNA sequence at a concentration of $0.1 \mathrm{ng} / \mu \mathrm{l}$ or $0.5 \mathrm{ng} / \mu \mathrm{l}$. We then measured miRNA levels in cell lysates by quantitative real time PCR (qPCR), 72 hours (hrs) post transfection (Sup. Figure 1a). Transfected miR-142 repressed the expression of a known target, Cofilin $2(\mathrm{Cfl} 2)^{35}$ by $70 \%$ after 72 hrs., relative to untransfected or scrambled mimic controls (Sup. Figure $1 \mathrm{~b}$ ). Thus, an exogenous, transfected, miRNA mimic functionally silences endogenous targets in primary mouse motor neurons.

Then we selected nine different miRNA candidates for investigation, including miR-9 ${ }^{36}, \mathrm{miR}-29^{37}, \mathrm{miR}-135^{38}$, miR-138 $8^{39}$, miR-30 $c^{40}$, miR- $124^{41}$, miR-218 ${ }^{10-12}$, miR-10a ${ }^{42}$ and miR-206 ${ }^{6}$. A qPCR study revealed that the synthetic mimics upregulated miRNA expression, $72 \mathrm{hrs}$ after transfection (Fig. 1a).

We then cultured primary motor neurons in 384-multiwell plates, transfected miRNA mimics and employed an ImageXpress Micro XLS Widefield High-Content Analysis System for measuring cell numbers, neurite outgrowth and number of branches per cell (Fig. $1 \mathrm{~b}$ and Sup. Figure 2). By applying Sodium Arsenite $\left(\mathrm{NaAsO}_{2}, 15\right.$ or 30 or $60 \mu \mathrm{M}$, for 60 minutes), a chemical that induces mitochondrial oxidative stress and leads to neurite outgrowth abnormalities ${ }^{43,44}$, we demonstrated the system's ability to sense morphometric abnormalities (Fig. 1c).

We tested the impact of the nine different miRNA candidates on neuronal morphology. Under all experimental conditions, cell numbers were unchanged, indicating that there was no cell death. While most miRNAs tested did not alter neuronal morphology, miR-124 overexpression caused a significant decrease in axon outgrowth and in the number of branches (Fig. 1d). 
a

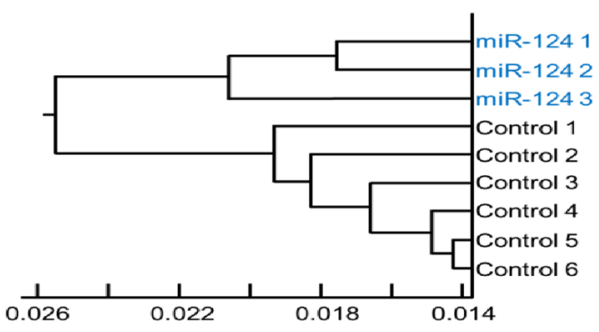

Correlative distance b

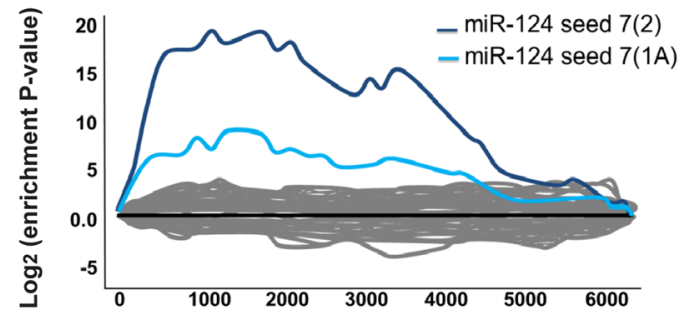

3'UTRs sorted from downregulated to upregulated

C

\begin{tabular}{|l|c|}
\hline Gene Ontology Term & Gene Count \\
\hline Organelle envelope & 27 \\
\hline Envelope & 27 \\
\hline Mitochondrial part & 24 \\
\hline Organelle inner membrane & 23 \\
\hline Mitochondrial membrane & 22 \\
\hline Mitochondrial envelope & 22 \\
\hline Mitochondrial inner membrane & 21 \\
\hline
\end{tabular}

Figure 2. Unbiased bioinformatics analysis of miR-124 targets. Next generation sequencing was performed on RNA extracted from primary motor neurons transfected with either miR-124 mimics $(\mathrm{N}=3)$ or control mimics $(\mathrm{N}=6)$. (a) A dendrogram depicting the hierarchical clustering of Pearson correlation coefficients for global gene expression from miR-124 overexpressing motor neurons (blue) and controls (black). (b) Enrichment landscape plot for all $8767 \mathrm{mer}$ motifs complementary to canonical mouse miRNA seed regions, gained by Sylamer analysis ${ }^{45}$. Sorted 6500 mRNAs expressed in primary motor neurons, ranked from down- to up-regulated after overexpressing of miR-124, or control mimic. Assessment of over- and under-represented miRNA recognition sequences (seed-matches) for all known miRNAs, identified two enriched motifs, both matching the miR-124 'seed' sequence (blue, 7mer-2; light blue, 7mer-1A). (c) Top seven terms (by gene count) from gene ontology (GO) analysis on all mRNAs significantly up- or down- regulated following miR-124 overexpression (corrected P-value $<0.05$ ), using database for annotation, visualization and integrated discovery (DAVID) software ${ }^{46}$. This analysis revealed an enrichment for mitochondrial-related genes and is further described in Table 1.

To uncover the underlying molecular mechanism responsible for miR-124 activity, we performed transcriptome profiling, using next generation sequencing (NGS). Total RNA was extracted from primary motor neurons and $3^{\prime}$ cDNA libraries were constructed and sequenced. Hierarchical clustering analysis of mRNA expression depicted a unique expression profile for neurons that overexpressed miR-124, which was distinguishable from cells transfected with scrambled control oligos (dendrogram of Pearson correlation coefficient, Fig. 2a).

Sylamer analysis ${ }^{45}$ of 6500 expressed mRNAs, from neurons expressing scrambled control mimics or miR-124, uncovered two enriched motifs, which matched the miR-124 'seed' sequence. However, such enrichment was not evident for any other miRNA gene (Fig. 2b). We conclude that miR-124 overexpression had a widespread and specific impact on motor neuron mRNA expression profile.

Gene ontology (GO) analysis was carried out for approximately 1100 mRNAs that were significantly up- or downregulated, following miR-124 overexpression (corrected P-value $<0.05$ ), using DAVID ${ }^{46}$. Intriguingly, many of the gene ontology terms highlighted the potential relevance of mitochondria-related structure or function in response to miR-124 (Table 1, Fig. 2c). We therefore further hypothesized that miR-124 plays a role in the regulation of mitochondrial functions.

We studied mitochondria function and intracellular position by employing Mitotracker Deep Red FM, a mitochondrion-selective dye that accumulates in active mitochondria. We also used in parallel Tetramethylrhodamine ethyl ester (TMRE), which is a fluorescent indicator of mitochondria membrane potential. Both TMRE and Mitotracker signals were dampened by the activity of Oligomycin $\mathrm{A}^{47}$, a benchmark inhibitor of mitochondrial ATP synthase. Interestingly, miR-124 overexpression inhibited TMRE and Mitotracker signals reminiscent of Oligomycin A, leading us to conclude that miR-124 regulates mitochondrial function (Fig. 3).

Confocal immunofluorescence of the mitochondrial marker, ATP5A, and an ultrastructural study with transmission electron microscopy (TEM), revealed depletion of mitochondria in primary motor neuron axons, which overexpressed miR-124, relative to controls. (Sup. Figures 3, 4). Therefore, miR-124 regulates mitochondria position and activity in motor neurons.

To characterize the targets and pathways that are regulated by miR-124, we intersected the list of mRNAs that were repressed more than twofold by miR-124 overexpression in the NGS data, with the list of predicted miR-124 targets (TargetScan ${ }^{48}$ ). Three genes that harbor conserved miR-124 binding sites, were also downregulated more 


\begin{tabular}{|c|c|c|}
\hline Gene Ontology term & $\begin{array}{l}\text { genes } \\
\text { count }\end{array}$ & P-value \\
\hline Organelle envelope & 27 & $1.80 \mathrm{E}-05$ \\
\hline Envelope & 27 & $1.90 \mathrm{E}-05$ \\
\hline Mitochondrial part & 24 & $2.30 \mathrm{E}-04$ \\
\hline Organelle inner membrane & 23 & $1.80 \mathrm{E}-07$ \\
\hline Mitochondrial membrane & 22 & $1.00 \mathrm{E}-05$ \\
\hline Mitochondrial envelope & 22 & $2.50 \mathrm{E}-05$ \\
\hline Mitochondrial inner membrane & 21 & $1.30 \mathrm{E}-06$ \\
\hline Inorganic cation transmembrane transporter activity & 16 & $8.40 \mathrm{E}-09$ \\
\hline Monovalent inorganic cation transmembrane transporter activity & 14 & $4.70 \mathrm{E}-09$ \\
\hline Hydrogen ion transmembrane transporter activity & 13 & $2.40 \mathrm{E}-08$ \\
\hline Redox-active center & 8 & $4.60 \mathrm{E}-06$ \\
\hline Cell redox homeostasis & 8 & $1.00 \mathrm{E}-04$ \\
\hline Thioredoxin-like & 6 & $3.40 \mathrm{E}-04$ \\
\hline Thioredoxin-like & 6 & $3.40 \mathrm{E}-04$ \\
\hline Disulphide isomerase & 5 & $1.20 \mathrm{E}-06$ \\
\hline Domain:Thioredoxin 2 & 5 & $9.30 \mathrm{E}-06$ \\
\hline Domain:Thioredoxin 1 & 5 & $9.30 \mathrm{E}-06$ \\
\hline Thioredoxin-like subdomain & 5 & $2.50 \mathrm{E}-05$ \\
\hline Thioredoxin, conserved site & 5 & $7.80 \mathrm{E}-04$ \\
\hline Thioredoxin domain & 5 & $9.10 \mathrm{E}-04$ \\
\hline Oxidoreductase activity, acting on heme group of donors, oxygen as acceptor & 5 & $6.90 \mathrm{E}-04$ \\
\hline Oxidoreductase activity, acting on heme group of donors & 5 & $6.90 \mathrm{E}-04$ \\
\hline Cytochrome-c oxidase activity & 5 & $6.90 \mathrm{E}-04$ \\
\hline Heme-copper terminal oxidase activity & 5 & $6.90 \mathrm{E}-04$ \\
\hline PIRSF001487:protein disulfide-isomerase & 4 & $1.90 \mathrm{E}-04$ \\
\hline Protein disulfide isomerase activity & 4 & $3.00 \mathrm{E}-04$ \\
\hline Intramolecular oxidoreductase activity, transposing S-S bonds & 4 & $3.00 \mathrm{E}-04$ \\
\hline Intramolecular oxidoreductase activity, interconverting keto- and enol-groups & 4 & $4.50 \mathrm{E}-04$ \\
\hline Intramolecular oxidoreductase activity & 4 & $3.10 \mathrm{E}-02$ \\
\hline Protein disulphide isomerase & 3 & $1.70 \mathrm{E}-03$ \\
\hline
\end{tabular}

Table 1. Gene ontology (GO) terms for changes in motor neuron mRNA expression after miR-124 overexpression. Top 30 Gene ontology (GO) term analysis of up- or downregulated mRNAs after miR-124 overexpression (corrected P-value $<0.05$ ). Table columns include DAVID ${ }^{46}$ term names, number of genes counted in each category and $\mathrm{p}$-value for the statistical significance of the category. There is noticeable overrepresentation of gene ontology terms related to mitochondria structure or function in response to miR-124.

than twofold by miR-124 overexpression, namely, Polypyrimidine Tract Binding Protein 1 (Ptbp1, MGI:97791), Midkine (Mdk, MGI:96949) and Vimentin (Vim, MGI:98932; Fig. 4a). qPCR study validated that miR-124 overexpression inhibited Vim to $\sim 1 / 3$ its levels compared with control cells that were treated with scrambled oligos. Ptbp1, an established miR-124 target ${ }^{49}$, and Mdk were downregulated to $\sim 1 / 2$ their expression level (Fig. $4 \mathrm{~b}$ ).

Interestingly, Vim is a known regulator of mitochondria localization and activity ${ }^{31,32}$, with miR-124 binding sites that are conserved across several vertebrate species (Fig. 4c). Furthermore, we identified molecular evidence for direct interactions of miR-124 with the target Vim, in Argonaute CLIP studies ${ }^{50,51}$ and a Vim $3^{\prime}$ UTR reporter was inhibited by miR-124 mimics in hepatocellular carcinoma cells ${ }^{52}$.

We sought to inhibit Vim independently of miR-124, and test its effect on cell morphology and mitochondria activity. Lentiviral transduction of primary motor neurons was very efficient (Sup. Figure 5) and allowed us to effectively knockdown Vim by shRNA ${ }^{53}$. Vim shRNA reduced Vim mRNA and protein levels (Fig. 4d,e, Sup. Figure 6a). Accordingly, high content image analysis of Vim knockdown depicted reduction in neurite outgrowth and branching (Fig. 4f), recapitulating miR-124 activity. In addition, Vim knockdown resulted in inhibition of mitochondria activity in axons, but not in the soma (Fig. 4g-i). Differences in the effect of miR-124 on soma may be the result of transfection efficiency. Intriguingly, miR-124 is primarily peri-nuclear ${ }^{54}$. Therefore, the soma compartment may be less amendable to manipulation, whereas, in the axon, where the miRNA is expressed at lower levels, the effects of miR-124 overexpression were consistent across all observations. In conclusion, Vim pheno-copies miR-124 functions, further suggesting that both genes are engaged in the same pathway.

Based on the above observations, we hypothesized that Vim is a novel effector of miR-124 in a pathway that regulates mitochondria function. Therefore, we tested whether upregulating Vim levels is sufficient for recovering mitochondrial activity. Doxycycline-induced expression of Vim, from a lentiviral vector that does not harbor the $3^{\prime} \mathrm{UTR}$ and hence is not inhibited by miR-124, upregulated Vim mRNA and protein levels (Fig. 5a,b, Sup. Figure 6b). Exogenous Vim was also sufficient to alleviate miR-124-dependent inhibition of mitochondrial 
a
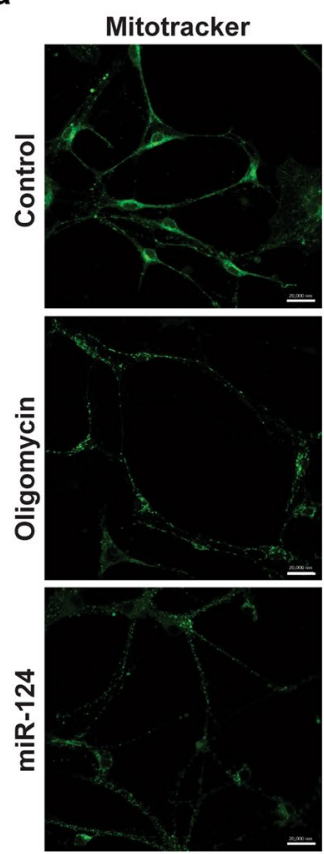
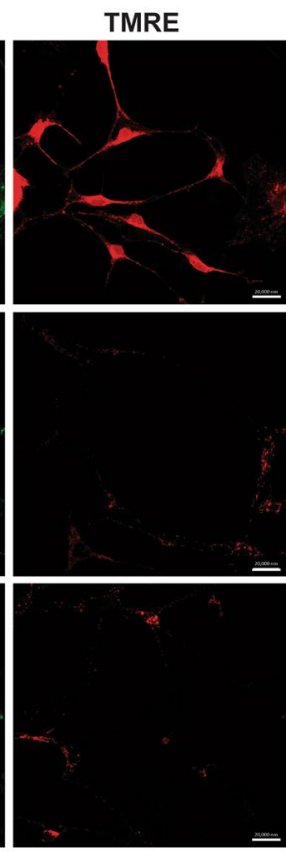

Merged + Hoechst
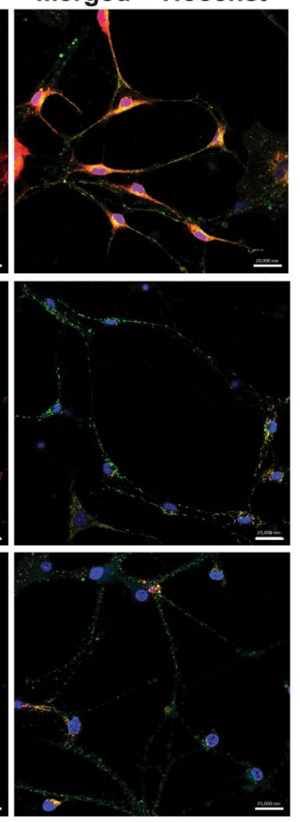

b

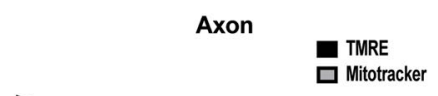

C

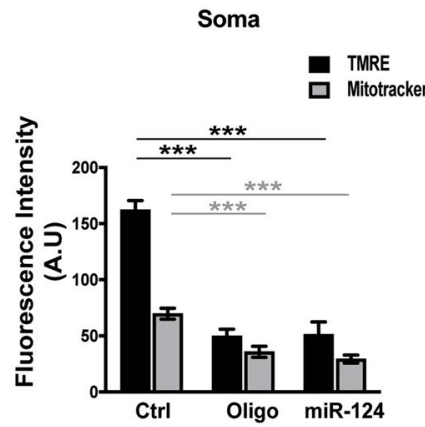

Figure 3. miR-124 overexpression impacts mitochondrial localization and function. (a) Confocal fluorescent micrographs of primary motor neurons, $72 \mathrm{hrs}$ post transfection with scrambled control or miR-124 mimics, stained with MitoTracker Deep Red FM (green) and TMRE (red) to depict functional mitochondria. Merged signal (yellow) relative to nuclei (blue, Hoechst), scale bars, $20 \mu \mathrm{m}$. Oligomycin A (1 $\mu \mathrm{M})$ or miR-124

overexpression diminished mitochondrial activity. Bar graphs quantification of fluorescent signal intensity in (b) axons and (c) soma. Quantification of 3 random positions per compartment; 9 neurons per condition; Data collected from three replicates and the study performed in $>$ four independent experimental repeats. Averages \pm SEM, one way ANOVA with post hock Neuman-Keuls. *P-value $<0.05, * * \mathrm{p}<0.01, * * * \mathrm{p}<0.001$.

function, relative to miR-124 alone (Fig. 5c-e). Furthermore, when counting the numbers of mitochondria in axons, we observed rescue of axonal mitochondria occupancy by exogenous Vim (Fig. $5 \mathrm{f}-\mathrm{h}$; We also noted some effect without Dox that is probably due to leaky Vim expression).

Finally, we used live imaging microscopy to test if the new miR-124-Vim axis regulates mitochondria motility in axons. We identified two subtypes of motile mitochondria: mitochondria that were continuously running in an uninterrupted manner along the axon over a distance of $>10 \mu \mathrm{m}$ at average speed $>0.2 \mu \mathrm{m} / \mathrm{sec}$., or mitochondria that displayed intermittent pauses in the same location for $\geq 3$ frames in succession. Intriguingly, miR- 124 caused mitochondria to pause more frequently on anterograde route, than in control samples, but did not affect retrograde transport. This may explain the re-distribution of mitochondria after overexpression of miR-124 and relative axonal depletion. Exogenous Vim expression rescued this phenotype and normalized mitochondria running/pausing dynamics (Fig. 6a-c). Furthermore, measurements of pause duration depicted asymmetry in anterograde vs. retrograde (Fig. 6d,e). We noted some effect without Dox that is probably due to leaky Vim expression. Parameters of run length and mean speed, were not changed by miR-124 and Vim (Sup. Figure 7). Therefore, anterograde mitochondria transport is influenced by the number of mitochondria pausing and the typical time interval spent resting during their run. We conclude that the mechanism for control of mitochondria running/pausing propensities in motor neurons involves Vim and miR-124 in a fashion affecting anterograde but not retrograde transport.

Taken together, we characterized a novel pathway in motor neurons downstream of miR-124 in regulation of mitochondria dynamics, distribution and activity. In this pathway, Vim, functions as an important effector of miR-124, revealing a surprising mechanism for controlling energy metabolism in motor neurons by neuronal miRNAs and intermediate filaments.

\section{Discussion}

In the current study, we employed high content image analysis, next generation sequencing and molecular approaches for discovery of a new pathway that is affecting motor neuron axon morphology and mitochondria homeostasis. An initial screen led us to focus on miR-124, which was the only miRNA that exhibited abnormal axonal morphology, out of nine miRNAs that were tested. miR-124 is one of the most abundant miRNA in many neuronal subtypes and is conserved from insects to mammals. miR-124 drives neuronal differentiation, promoting neuroblasts cell-cycle exit ${ }^{41,55}$, neuron-specific alternative splicing and chromatin remodeling via silencing of Ptbp $11^{49}$ and Actin like 6A/BAF53a ${ }^{56}$, respectively. Furthermore, miR-124 levels remain high in postmitotic neurons, suggesting that it plays a role also in maintenance of the differentiated state of neurons. However, miR124 roles in motor neuron were not thoroughly investigated. 
a

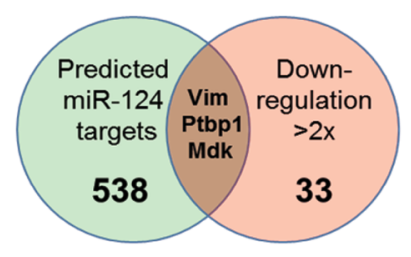

b

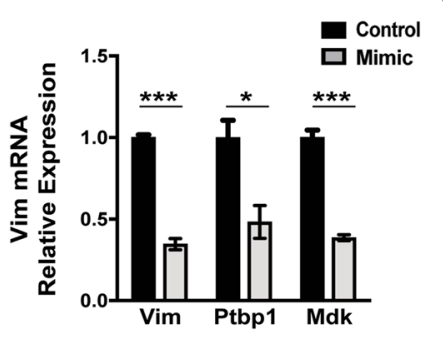

C

Human C-AGC-UUU-CAAGUGCC-UUU-CUGCA--GChimp C-AGC-UUU-CAAGUGCC-UUU-CUGCA-G Mouse C-AGC-UUU-CAAGUGEC-UUUACUGCA G Elephant C-AGC-UUU-CAAGUGCC-UUU-CUGCA-G Chicken C-AGC-UUU-CAAGUGCC-UUU-CUCCA-G Lizard C-CGC-UUU-CAAGUGCC-UUU-CUGCA-G d

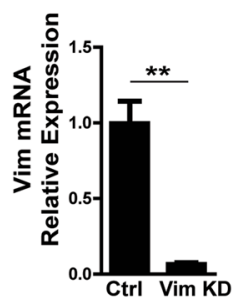

g

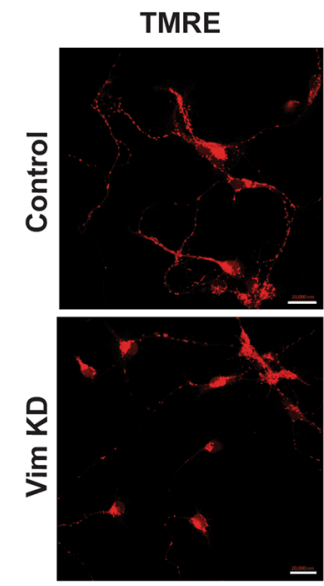

e
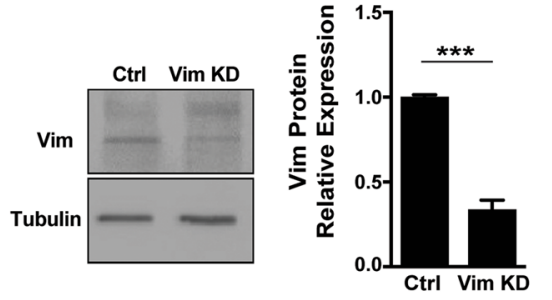

f

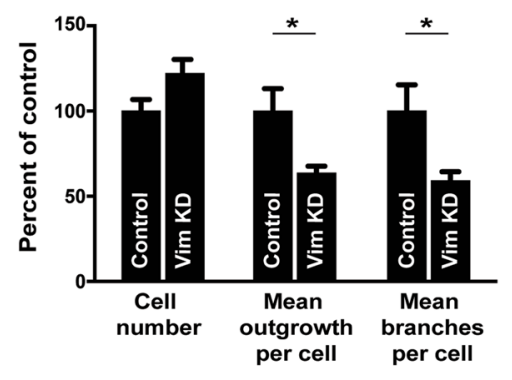

h

TMRE + Hoescht
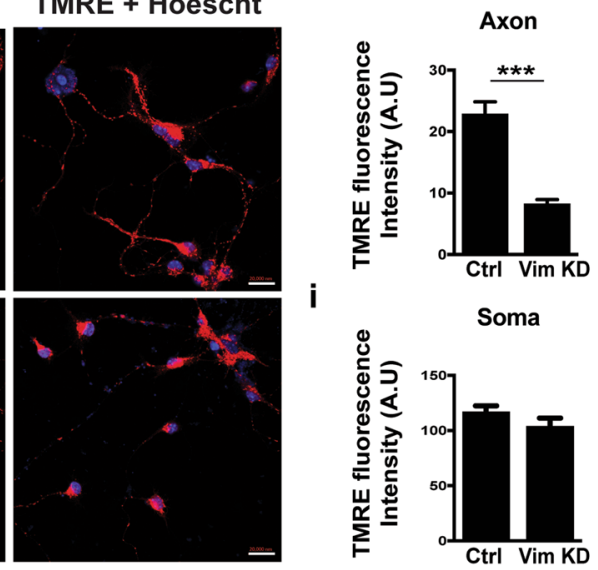

Figure 4. Vimentin regulates neuron morphology and mitochondria function (a) Venn diagram of predicted miR-124 targets (TargetScan) and transcripts that were experimentally repressed $>2$ fold by miR-124 overexpression in primary motor neurons, relative to control conditions. (b) qPCR analysis of the three mRNAs that obeyed both criterions: Vim, Ptbp1 and Mdk, in primary motor neurons transfected with miR-124 oligos $(\mathrm{N}=3)$, relative to controls $(\mathrm{N}=3)$. (c) miR-124 recognition element at Vim 3'UTR is conserved in vertebrate species. Knockdown of Vim by shRNA lentiviruses ${ }^{53}$ downregulated (d) Vim mRNA and (e) VIM protein expression, relative to non-targeting shRNA controls. A full view of the Western blot is in Sup. Figure 6. (f) High content image analysis of cell numbers, mean axonal outgrowth per cell and mean number of branches per cell. 100 Tuj $1+$ neurons quantified per field, 8 fields/well, 6 replicates per treatment. Data collected from $>24,000$ Tuj1+ neurons per treatment. (g) Confocal fluorescent micrographs of primary motor neurons, $72 \mathrm{hrs}$. post transduction with scrambled-shRNA or Vim-shRNA, stained with TMRE (red) and nuclei (blue, Hoechst). Transduction efficacy $>80 \%$. Scale bars, $20 \mu \mathrm{m}$. Bar graphs quantification of fluorescent signal intensity in (h) axons and (i) soma. Quantification of 3 random positions per compartment; 9 neurons per condition. Data from three replicates and the study performed in three independent experimental repeats. All graphs present averages \pm SEM, Student's t-test. $* \mathrm{P}$-value $<0.05, * * \mathrm{p}<0.01, * * * \mathrm{p}<0.001$

We demonstrated that miR-124 regulates mitochondrial activity and localization. The cluster of mitochondrial genes that responded to miR-124 overexpression was not particularly enriched in direct targets of miR-124, suggesting indirect regulation. The intermediate filament Vim is a key effector of miR-124 upstream of mitochondria function and localization. Vim knockdown by other means, pheno-copied miR-124 overexpression and an exogenous Vim that does not harbor miR-124 binding sites, rescued the mitochondrial phenotype.

Precise mitochondria localization is critical for maintaining energy and calcium homeostasis in neurons. Appropriate mitochondria localization is essential for neurite outgrowth ${ }^{57-59}$. Minin et al., have shown that Vim regulates mitochondria activity and motility ${ }^{31,32,60}$ in other cell types, which is consistent with the discovery of a new miR-124 - Vim axis for unidirectional control of mitochondria transport in axons. miR-124 and Vim 
a

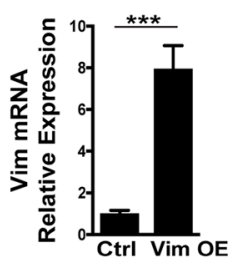

b
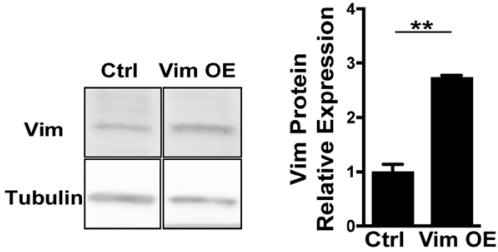

C
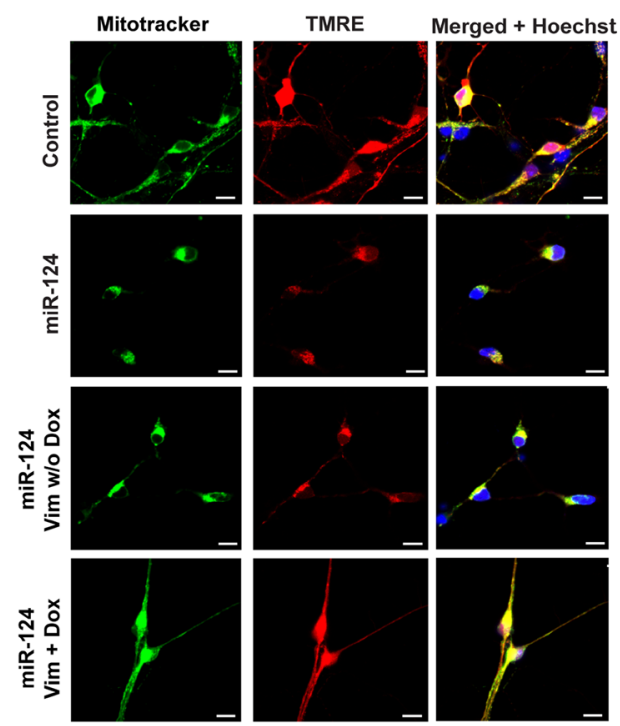

f

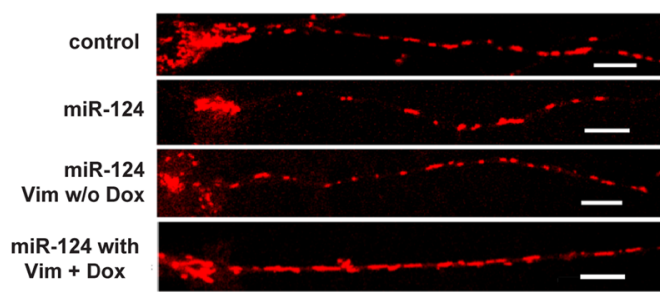

h

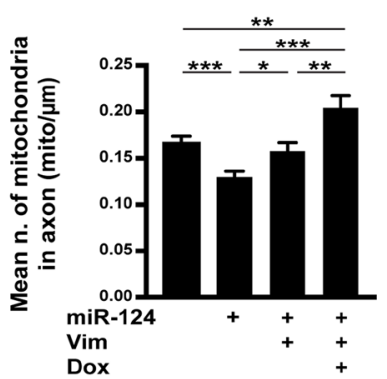

d

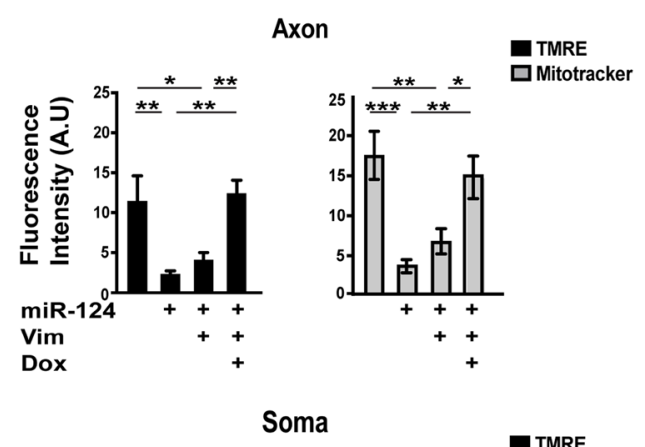

Mitotracke
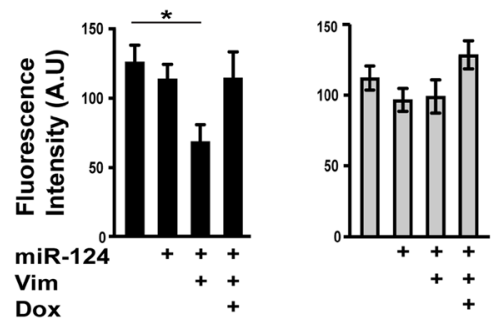

g

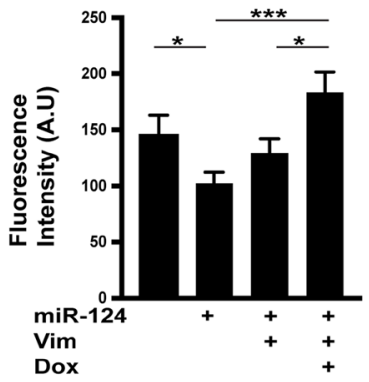

Figure 5. miR-124 regulates mitochondria activity via Vimentin. Primary motor neurons of control, miR-124 overexpression alone or transduced in addition with lentiviral vector for Doxycyclin-dependent expression of Vim ( $>80 \%$ transduction efficacy, transduction $24 \mathrm{hrs}$. before transfection of miRNA mimics), without or with the chemical inducer (Dox). (a) Vim mRNA and (b) VIM protein expression without or with Dox. Three experimental repeats, Averages \pm SEM, Student's $t$-test. A full view of the Western blot is in Sup. Figure 6. (c) Confocal fluorescent micrographs of primary motor neurons, stained with MitoTracker (green) and TMRE (red), merged signal (yellow) and nuclei (blue, Hoechst). Scale bars, $20 \mu \mathrm{m}$. Bar graphs quantification of fluorescent signal intensity in (d) axons and (e) soma. Quantification of three random positions per compartment; 9 neurons per condition; Data from three replicates and the study performed in three independent experimental repeats. Averages \pm SEM, ANOVA + Duncan's new multiple range test (MRT) $* \mathrm{P}$-value $<0.05$, ** $\mathrm{p}<0.01$, *** $\mathrm{p}<0.001$. (f) Snapshot from mitochondria live imaging along axons of primary motor neurons. Bar graphs quantification of $(\mathbf{g})$ axonal mitochondria density $/ \mu \mathrm{m}$ and $(\mathbf{h})$ mean TMRE intensity in control axons $(\mathrm{n}=48)$, miR-124 overexpression alone $(\mathrm{n}=52)$, miR-124 and Vim without $\operatorname{Dox}(\mathrm{n}=34)$ or with $\operatorname{Dox}(\mathrm{n}=34)$. Averages \pm SEM, Student's $t$-test, P-value $* \mathrm{p}<0.05, * * \mathrm{p}<0.01$. 
a

Control
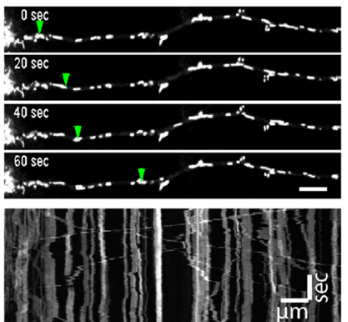

Anterograde $\longrightarrow$

b

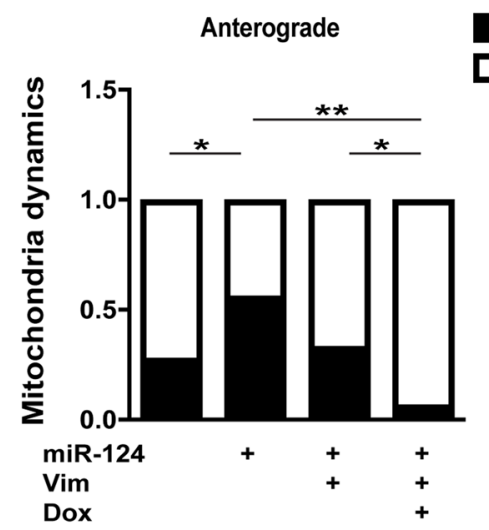

d

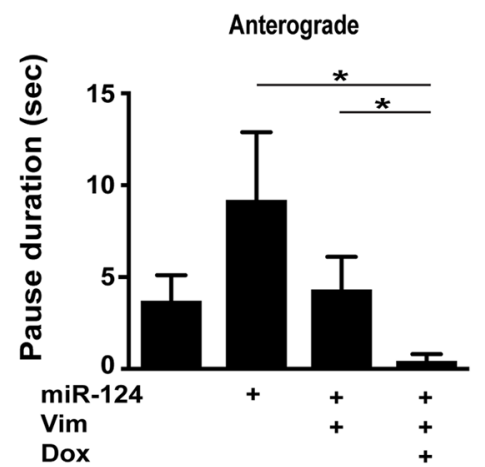

C

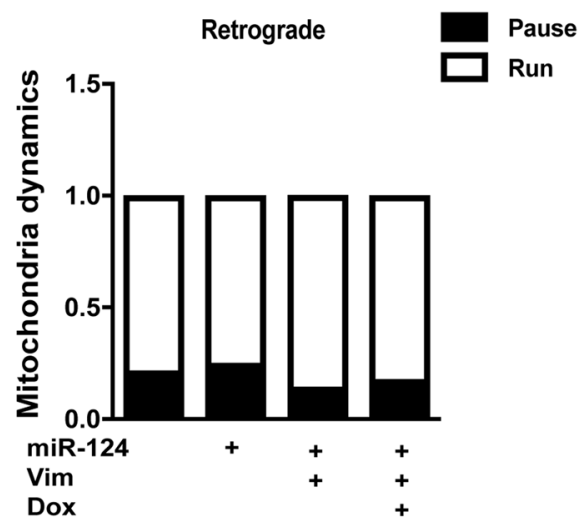

e

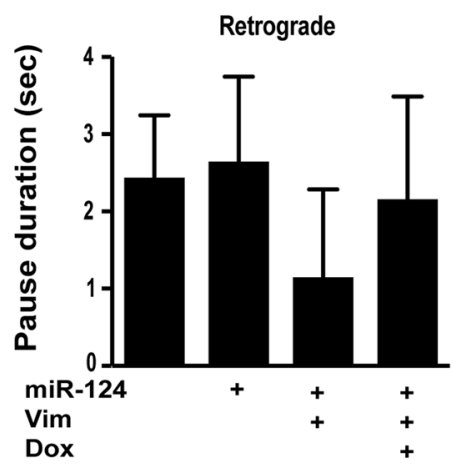

Figure 6. miR-124 and Vimentin co-regulate mitochondria motility in primary motor neurons. Primary motor neurons of control, miR-124 overexpression alone or transduced in addition with lentiviral vector for Doxycycline-dependent expression of Vim ( $>80 \%$ transduction efficacy, performed 24 hrs. before transfection of miRNA mimics), without or with the chemical inducer (Dox). (a) Representative captures from mitochondria live imaging and motor axons kymographs, depicting anterograde movement. Green arrows indicate the movement of one representative mitochondrion. Running mitochondria were defined by moving a distance of $>10 \mu \mathrm{m}$ at average speed $>0.2 \mu \mathrm{m} / \mathrm{sec}$. Paused mitochondria are a subpopulation of the running mitochondria, which paused in the same location for $\geq 3$ frames in succession. Proportion of pausing and continuously running mitochondria in (b) anterograde or (c) retrograde directions. Horizontal scale bar $10 \mu \mathrm{m}$. Vertical scale bar -60 seconds. Test of proportion statistics, ${ }^{*} \mathrm{p}<0.05$, $* * \mathrm{p}<0.01$. (d) Anterograde or (e) retrograde measurement of pause duration (sec). Averages \pm SEM, Student's $t$-test, P-value *p $<0.05$.

asymmetric action is further evocative of kinesins and dynein motor proteins that reciprocally serve anterograde and retrograde transport. However, a direct molecular link to the classic kinesin/dynein system is still missing.

miRNA dysregulation ${ }^{7,61-63}$ and mitochondrial impairments ${ }^{63-65}$, are repeatedly observed in ALS. Our analysis reveals that miR-124 overexpression may be disadvantageous to primary motor neurons, in accordance with reported miR-124 upregulation in late ALS stages in mouse brains ${ }^{61}$ and with injurious miR-124 overexpression in adult hippocampus/prefrontal cortex ${ }^{66}$. In summary, we propose that miR-124 expression levels should be 
tightly kept within defined margins and that a novel miR-124 - Vim pathway reveals a mechanism, by which miRNAs regulate of axonal mitochondria transport.

\section{Materials and Methods}

Primary motor neuron culture. All experiments were performed in accordance with relevant guidelines and were approved by the Institutional Animal Care and Use Committee (IACUC) at Weizmann Institute of Science. Primary motor neurons were isolated and cultured as described ${ }^{34}$ with the following modification: spinal cords were dissected from ICR mouse embryos at embryonic day 13.5 (E13.5). Motor neurons were dissociated with papain $(2 \mathrm{mg} / \mathrm{ml}$, Sigma), separated through Optiprep gradient (Sigma) and seeded either on $13 \mathrm{~mm}$ coverslips (200,000 cells/coverslip, Thermo scientific) or on 384 multiwell plates (7500 cells/well, Griener bio-one, cat\# 781091) using a liquid handling device (GNF Systems), pre-coated with poly-ornithine ( $3 \mu \mathrm{g} / \mathrm{ml})$ or poly $\mathrm{L}$ Lysine (Sigma P4707, 0.002\% in Borate buffer 0.1 M ph 8.5, Sigma) and then Laminin ( $3 \mu \mathrm{g} / \mathrm{ml}$, Gibco | Thermo Fisher Scientific). Motor neurons were cultured with Neurobasal (Gibco | Thermo Fisher Scientific)/B27 (Gibco | Thermo Fisher Scientific) medium supplemented with $2 \%$ horse serum (Sigma), and $1 \mathrm{ng} / \mathrm{ml} \mathrm{CNTF,} 1 \mathrm{ng} / \mathrm{ml}$ GDNF (Peprotech) at $37^{\circ} \mathrm{C}$. For live imaging motor neurons were isolated as in ${ }^{67,68}$.

miRNA mimics transfection. miRNA mimics, were dsRNA oligonucleotides (Integrated DNA Technologies, Inc.), as described in Table 2. dsRNA encapsulated in Neuro ${ }^{\mathrm{TM}}$ nanoparticles (Precision NanoSystems, Inc. $)^{33}$. Mimics $(0.5 \mathrm{ng} / \mu \mathrm{l})$ were transfected $24 \mathrm{hrs}$ after seeding of primary motor neurons manualy or by using Bravo automated liquid handling robot.

Lentiviruses. Vimentin shRNA lentiviruses were described $\mathrm{in}^{53}$. Cells were transduced simultaneously with both versions of sh-Vim lentiviruses, $24 \mathrm{hrs}$ post seeding, and downstream analyses performed at $72 \mathrm{hrs}$. For overexpression, human Vimentin coding region, was subcloned into FuW-TetO lentiviral vector downstream of tetracyclin response element. A mix of two lentiviruses, one for the expression of Vimentin and the other with FUW-m2rtTA (reverse tetracycline-controlled transactivator), were transduced at MOI $=5$, one hour after primary motor neuron seeding. Doxycycline ( $1 \mu \mathrm{g} / \mathrm{ml}$, Sigma) was added at $24 \mathrm{hrs}, 72 \mathrm{hrs}$ post infection, and downstream analyses performed at $96 \mathrm{hrs}$.

Mitochnodria assays. The following chemicals were added, when mentioned: MitoTracker ${ }^{\circledR}$ Deep Red 633 (M22426, Molecular Probes, 50 nM) tetramethylrhodamine, ethyl ester (TMRE, $200 \mathrm{nM}$ ) Hoechst (Sigma, $1 \mu \mathrm{g} /$ $\mathrm{mL}) 30 \mathrm{~min} 37^{\circ} \mathrm{C}$. Oligomycin A (Sigma, $1 \mu \mathrm{M}$ ) for $15 \mathrm{~min}$, in $37^{\circ} \mathrm{C}$ and analyzed $24 \mathrm{hrs}$. afterwards.

Immunostaining and Western Blot analysis. Cells were rinsed with phosphate buffered saline (PBS) 3 time manually or using Biotek EL406 washer/dispenser for automated 384-well microplates, fixed with $4 \%$ formaldehyde (ChemCruz ${ }^{\mathrm{TM}}$ ) for $15 \mathrm{~min}$, permeabilized and blocked in $0.1 \%$ Triton X-100 (Sigma), $2 \%$ BSA for 20/60 min for 384/24 well plate setup, respectively. Incubation with anti-neuronal Class III beta Tubulin (Tuj1) (1:1000, MRB-435P, Covance, 1.5 hrs) and/or anti-ATP5A, (1:500,ab14748, Abcam) or antiMicrotubule-associated protein 2 (MAP2) (1:500, sc-20172) was followed by anti Cy2- conjugated donkey anti-rabbit IgG (1:200, Jackson ImmunoResearch, $1 \mathrm{hr})$, nuclear staining with 4'6-Diamidino-2-phenylindole dihydrochloride (DAPI, $1 \mu \mathrm{g} / \mathrm{ml}$,sigma, $5 \mathrm{~min}$.) and 3 cycles of PBS rinsing. For Western blot studies, $30 \mu \mathrm{g}$ protein extracts were denatured by boiling in $\times 5$ sample buffer $(60 \mathrm{mM}$ Tris- $\mathrm{HCl} \mathrm{pH} 6.8,25 \%$ glycerol, $2 \%$ SDS, $14.4 \mathrm{mM} \beta$-mercaptoethanol, $0.1 \%$ bromophenol blue) for $5 \mathrm{~min}$., resolved by $8 \%$ SDS-PAGE, $100-120 \mathrm{~V}, 70 \mathrm{~min}$. and transferred to nitrocellulose membrane (Whatmann, 10401383) at $250 \mathrm{~mA}, 70 \mathrm{~min}$. Membranes were stained with Ponceau (Sigma-Aldrich, P7170), blocked for $60 \mathrm{~min}$. at Room Temperature (RT) with 5\% milk, 0.05\% TWEEN-20 and incubated, rocking, with primary antibodies O.N. at $4{ }^{\circ} \mathrm{C}$, namely, monoclonal mouse anti-Vim supernatant (a gift from Benny Geiger, Weizmann Institute of Science) or monoclonal mouse anti-alpha-Tubulin, (DM1A SigmaT9026 1:2000 in 5\% Bovine Serum Albumin, 0.02\% sodium azide, 5 drops of phenol red in 0.05\% PBST). Membranes were washed $3 \times 5 \mathrm{~min}$., RT with $0.05 \%$ PBST and incubated $60 \mathrm{~min}$. with horseradish peroxidase (HRP)-conjugated anti-mouse secondary antibodies. Membranes were washed $3 \times 5$ min., processed with EZ-ECL Chemiluminescence detection kit for HRP (Biological Industries, 20-500-120) and visualized by ImageQuant $^{\mathrm{TM}}$ LAS 4000 (GE Healthcare Life Sciences). Vim densitometry values normalized to Tubulin in the same lane. Representative data in main Figs $4 \mathrm{e}$ and $5 \mathrm{~b}$ are depicted on blots, shown in full in Sup. Figure 6.

Imaging. For high content image analysis, eight/two micrographs taken in 24 /384 well plate setups, respectively, using automated fluorescence microscope (ImageXpress Micro and MetaXpress software, Molecular Devices). Motor neuron were defined by positive to Tuj1 staining (Alexa488, FITC channel) and nuclear stain (DAPI). Phenotypic parameters were quantified with relevant MetaXpress High-Content Image Acquisition modules (Neurite Outgrowth, MWCS). Confocal microscopy performed on Carl-Zeiss 710. Mitochondria Live imaging was performed on Nikon Eclipse Ti Spinning disc confocal with Yokogawa CSU X-1, 60X oil-immersion lens, Andor iXON3 EMCCD camera under controlled environment $\left(37^{\circ} \mathrm{C}, 5 \% \mathrm{CO} 2\right)$. Axonal transport analysis was carried by analysis of time-lapse images using image J or MATLAB, as in ${ }^{67,68}$. For transmission electron microscopy, motor neuron were prepared, following ${ }^{69}$ and electron micrographs were captured with a FEI Tecnai SPIRIT transmission electron microscope (FEI, Eidhoven, Netherlands), operated at $120 \mathrm{kV}$ and equipped with an EAGLE CCD Camera.

RNA analysis and Next Generation Sequencing. Total RNA was isolated with miRNeasy micro kit (Qiagen), assessed with Nano Drop ND-1000 Spectrophotometer (Peqlab) and reverse transcribed to cDNA. qPCR, performed with SYBR Green (Thermo Fisher Scientific or Qiagen). miRNA/mRNA levels were normalized to U6/hypoxanthine phosphoribosyltransferase 1 (Hprt), respectively. Primer sequences are described in Table 2. 


\begin{tabular}{|c|c|c|}
\hline \multicolumn{3}{|c|}{ miRNA mimics, designed as dsRNA oligonucleotides $r-$ RNA bases; $\mathbf{r}_{-}$* - Phosphorothioated RNA base; m- 2' O-methyl RNA base } \\
\hline & Anti- sense mimic & Sense mimic \\
\hline miR-142-3p & rU*rG*rUrArGrUrGrUrUrUrCrCrUrArCrUrUrUrArUmGmGrA & 5SpC3/rUmCrCmArUmArAmArGmUrAmGrGmArAmArCmArCmUrAmCA \\
\hline miR-9-5p & rU*rC*rUrUrUrGrGrUrUrArUrCrUrArGrCrUrGrUrAmUmGrA & mAmUrAmCrAmGrCmUrAmGrAmUrAmArCmCrAmArAmGA \\
\hline miR-10a-5p & rU*rA*rCrCrCrUrGrUrArGrArUrCrCrGrArArUrUrUmGmUrG & mCmArAmArUmUrCmGrGmArUmCrUmArCmArGmGrGmUA \\
\hline miR-29a-3p & rU*rA*rGrCrArCrCrArUrCrUrGrArArArUrCrGrGmUmUrA & mAmCrCmGrAmUrUmUrCmArGmArUmGrGmUrGmCrUA \\
\hline miR-30c-5p & rU*rG*rUrArArArCrArUrCrCrUrArCrArCrUrCrUrCmAmGrC & mUmGrAmGrAmGrUmGrUmArGmGrAmUrGmUrUmUrAmCA \\
\hline miR-124-3p & rU*rA*rArGrGrCrArCrGrCrGrGrUrGrArArUmGmCrC & mCmArUmUrCmArCmCrGmCrGmUrGmCrCmUrUA \\
\hline miR-135a-5p & rU*rA*rUrGrGrCrUrUrUrUrUrArUrUrCrCrUrArUrGmUmGrA & mAmCrAmUrAmGrGmArAmUrAmArAmArAmGrCmCrAmUA \\
\hline miR-138-5p & rA*rG*rCrUrGrGrUrGrUrUrGrUrGrArArUrCrArGrGmCmCrG & mGmCrCmUrGmArUmUrCmArCmArAmCrAmCrCmArGmCU \\
\hline miR-206-3p & rU*rG*rGrArArUrGrUrArArGrGrArArGrUrGrUrGmUmGrG & mAmCrAmCrAmCrUmUrCmCrUmUrAmCrAmUrUmCrCA \\
\hline miR-218-5p & rU*rU*rGrUrGrCrUrUrGrArUrCrUrArArCrCrAmUmGrU & mAmUrGmGrUmUrAmGrAmUrCmArAmGrCmArCmAA \\
\hline Control & $\mathrm{rC}^{*} \mathrm{rG}^{*} \mathrm{rCrGrArCrUrArUrArCrGrCrGrCrArArUrArUmGmGrU}$ & rAmCrCmArUmArUmUrGmCrGmCrGmUrAmUrAmGrUmCrGmCG \\
\hline \multicolumn{3}{|c|}{ Quantitative real time PCR DNA primers } \\
\hline Gene & Forward & Reverse \\
\hline Cfl2 & TCTCGTCCCAGTGCCACCGA & ACTCCAGATGCCATAGTGCCCGC \\
\hline Hprt & CTGGTTAAGCAGTACAGCCCCAAA & TGGCCTGTATCCAACACTTCGAGA \\
\hline Vim & AAATGGCTCGTCACCTTCGT & AGAAATCCTGCTCTCCTCGC \\
\hline Ptbp1 & ATTCCGTGTGGTCACAGACA & GTCACTGGAAGGAGCTCAGG \\
\hline Mdk & CCAGGAGACCATCCGCG & TCCTTTTCCTTTCTTGGCTTTG \\
\hline $\mathrm{miR}-142-3 p$ & TGTAGTGTTTCCTACTTTATGGA & Universal primer (Qiagen) \\
\hline miR-9-5p & TCTTTGGTTATCTAGCTGTATGA & Universal primer (Qiagen) \\
\hline miR-10a-5p & TACCCTGTAGATCCGAATTTGTG & Universal primer (Qiagen) \\
\hline miR-29a-3p & TAGCACCATCTGAAATCGGTTA & Universal primer (Qiagen) \\
\hline miR-30c-5p & TGTAAACATCCTACACTCTCAGC & Universal primer (Qiagen) \\
\hline miR-124-3p & TAAGGCACGCGGTGAATGCC & Universal primer (Qiagen) \\
\hline miR-135a-5p & TATGGCTTTTATTCCTATGTGA & Universal primer (Qiagen) \\
\hline miR-138-5p & AGCTGGTGTTGTGAATCAGGCCG & Universal primer (Qiagen) \\
\hline miR-206-3p & TGGAATGTAAGGAAGTGTGTGG & Universal primer (Qiagen) \\
\hline miR-218-5p & TTGTGCTTGATCTAACCATGT & Universal primer (Qiagen) \\
\hline RNU6B (U6) & GATGACACGCAAATTCGTGAA & Universal primer (Qiagen) \\
\hline
\end{tabular}

Table 2. List of synthetic DNA and RNA oligos used in the study.

Library prep for NGS, following [46] and sequencing performed on Illumina 2500 at $50 \mathrm{bp}$ single read. Fasta files created (Illumina CASAVA-1.8.2 software) and mapped (TopHat2 version v2.0.10) ${ }^{70}$ against the mouse genome, build $\mathrm{mm} 9$. Approximately, $85-90 \%$ mapping rate was observed. Only uniquely mapped reads determined the number of reads per gene (HTSeq-count script $0.6 .1 \mathrm{p} 1)^{71}$. Differentially expressed genes, were determined by padj $<0.05$ and an absolute fold change $>2$ (DESeq. 2 package v1.4.5) $)^{72}$ and hierarchical clustering using Pearson dissimilarity and complete linkage was performed to explore gene expression patterns (Matlab 8.0.0.783).

Statistical analysis. Analysis was performed manually or with GraphPad Prism 6 for Student's $t$-test, test of proportion or one way ANOVA with post-hoc Newman-Keuls or one way ANOVA with Duncan's new multiple range test (MRT), as indicated. Results are given as mean \pm standard error of the mean (s.e.m). The null hypothesis was rejected at the 0.05 level $\left(^{*}\right), 0.01\left(^{* *}\right)$ or $0.001\left(^{* * *}\right)$. Non-significant values on statistical test are not mentioned in the figures. Gene Ontology analysis was performed using DAVID ${ }^{46}$.

\section{References}

1. Bartel, D. P. MicroRNAs: target recognition and regulatory functions. Cell 136, 215-233, https://doi.org/10.1016/j.cell.2009.01.002 (2009).

2. Kosik, K. S. The neuronal microRNA system. Nature reviews. Neuroscience 7, 911-920, https://doi.org/10.1038/nrn2037 (2006).

3. Schratt, G. microRNAs at the synapse. Nature reviews. Neuroscience 10, 842-849, https://doi.org/10.1038/nrn2763 (2009).

4. Haramati, S. et al. miRNA malfunction causes spinal motor neuron disease. Proceedings of the National Academy of Sciences of the United States of America 107, 13111-13116, https://doi.org/10.1073/pnas.1006151107 (2010).

5. Koval, E. D. et al. Method for widespread microRNA-155 inhibition prolongs survival in ALS-model mice. Human molecular genetics 22, 4127-4135, https://doi.org/10.1093/hmg/ddt261 (2013).

6. Williams, A. H. et al. MicroRNA-206 delays ALS progression and promotes regeneration of neuromuscular synapses in mice. Science 326, 1549-1554, https://doi.org/10.1126/science.1181046 (2009).

7. Emde, A. et al. Dysregulated miRNA biogenesis downstream of cellular stress and ALS-causing mutations: a new mechanism for ALS. EMBO J 34, 2633-2651, https://doi.org/10.15252/embj.201490493 (2015).

8. Greenamyre, J. T. Huntington's disease-making connections. The New England journal of medicine 356, 518-520, https://doi. org/10.1056/NEJMcibr067022 (2007)

9. De Felice, B. et al. miR-338-3p is over-expressed in blood, CFS, serum and spinal cord from sporadic amyotrophic lateral sclerosis patients. Neurogenetics 15, 243-253, https://doi.org/10.1007/s10048-014-0420-2 (2014). 
10. Amin, N. D. et al. Loss of motoneuron-specific microRNA-218 causes systemic neuromuscular failure. Science 350, 1525-1529, https://doi.org/10.1126/science.aad2509 (2015).

11. Hoye, M. L. et al. MicroRNA Profiling Reveals Marker of Motor Neuron Disease in ALS Models. The Journal of neuroscience: the official journal of the Society for Neuroscience 37, 5574-5586, https://doi.org/10.1523/JNEUROSCI.3582-16.2017 (2017).

12. Thiebes, K. P. et al. miR-218 is essential to establish motor neuron fate as a downstream effector of Isl1-Lhx3. Nat Commun 6, 7718, https://doi.org/10.1038/ncomms8718 (2015).

13. Filosto, M. et al. The role of mitochondria in neurodegenerative diseases. Journal of neurology 258, 1763-1774, https://doi. org/10.1007/s00415-011-6104-z (2011).

14. Lin, M. T. \& Beal, M. F. Mitochondrial dysfunction and oxidative stress in neurodegenerative diseases. Nature 443, 787-795, https:// doi.org/10.1038/nature05292 (2006).

15. Kitayama, H. et al. Human immunodeficiency virus type $1 \mathrm{Vpr}$ inhibits axonal outgrowth through induction of mitochondrial dysfunction. Journal of virology 82, 2528-2542, https://doi.org/10.1128/JVI.02094-07 (2008).

16. Hauptmann, S. et al. Mitochondrial dysfunction in sporadic and genetic Alzheimer's disease. Experimental gerontology 41, 668-673, https://doi.org/10.1016/j.exger.2006.03.012 (2006).

17. Pickrell, A. M. \& Youle, R. J. The Roles of PINK1, Parkin, and Mitochondrial Fidelity in Parkinson's Disease. Neuron 85, 257-273, https://doi.org/10.1016/j.neuron.2014.12.007 (2015)

18. Cozzolino, M. \& Carri, M. T. Mitochondrial dysfunction in ALS. Progress in neurobiology 97, 54-66, https://doi.org/10.1016/j. pneurobio.2011.06.003 (2012).

19. Sasaki, S. \& Iwata, M. Ultrastructural study of synapses in the anterior horn neurons of patients with amyotrophic lateral sclerosis. Neuroscience letters 204, 53-56 (1996).

20. Bandiera, S. et al. Nuclear outsourcing of RNA interference components to human mitochondria. PloS one 6, e20746, https://doi. org/10.1371/journal.pone.0020746 (2011).

21. Tomasetti, M., Neuzil, J. \& Dong, L. MicroRNAs as regulators of mitochondrial function: role in cancer suppression. Biochimica et biophysica acta 1840, 1441-1453, https://doi.org/10.1016/j.bbagen.2013.09.002 (2014).

22. Barrey, E. et al. Pre-microRNA and mature microRNA in human mitochondria. PloS one 6, e20220, https://doi.org/10.1371/journal. pone.0020220 (2011)

23. Huang, L. et al. Mitochondria associate with P-bodies and modulate microRNA-mediated RNA interference. The Journal of biological chemistry 286, 24219-24230, https://doi.org/10.1074/jbc.M111.240259 (2011).

24. Yoda, M. et al. ATP-dependent human RISC assembly pathways. Nature structural \& molecular biology 17, 17-23, https://doi. org/10.1038/nsmb.1733 (2010).

25. Zhang, X. et al. MicroRNA directly enhances mitochondrial translation during muscle differentiation. Cell 158, 607-619, https:// doi.org/10.1016/j.cell.2014.05.047 (2014).

26. Carrer, M. et al. Control of mitochondrial metabolism and systemic energy homeostasis by microRNAs 378 and $378^{*}$. Proceedings of the National Academy of Sciences of the United States of America 109, 15330-15335, https://doi.org/10.1073/pnas.1207605109 (2012).

27. Chan, S. Y. et al. MicroRNA-210 controls mitochondrial metabolism during hypoxia by repressing the iron-sulfur cluster assembly proteins ISCU1/2. Cell metabolism 10, 273-284, https://doi.org/10.1016/j.cmet.2009.08.015 (2009).

28. Aschrafi, A. et al. MicroRNA-338 regulates local cytochrome c oxidase IV mRNA levels and oxidative phosphorylation in the axons of sympathetic neurons. The Journal of neuroscience: the official journal of the Society for Neuroscience 28, 12581-12590, https://doi. org/10.1523/JNEUROSCI.3338-08.2008 (2008).

29. Aschrafi, A. et al. MicroRNA-338 regulates the axonal expression of multiple nuclear-encoded mitochondrial mRNAs encoding subunits of the oxidative phosphorylation machinery. Cellular and molecular life sciences: CMLS, https://doi.org/10.1007/s00018012-1064-8 (2012).

30. Kole, A. J., Swahari, V., Hammond, S. M. \& Deshmukh, M. miR-29b is activated during neuronal maturation and targets BH3-only genes to restrict apoptosis. Genes \& development 25, 125-130, https://doi.org/10.1101/gad.1975411 (2011).

31. Chernoivanenko, I. S., Matveeva, E. A., Gelfand, V. I., Goldman, R. D. \& Minin, A. A. Mitochondrial membrane potential is regulated by vimentin intermediate filaments. FASEB J 29, 820-827, https://doi.org/10.1096/fi.14-259903 (2015).

32. Nekrasova, O. E. et al. Vimentin intermediate filaments modulate the motility of mitochondria. Mol Biol Cell 22, 2282-2289, https:// doi.org/10.1091/mbc.E10-09-0766 (2011).

33. Rungta, R. L. et al. Lipid Nanoparticle Delivery of siRNA to Silence Neuronal Gene Expression in the Brain. Molecular therapy. Nucleic acids 2, e136, https://doi.org/10.1038/mtna.2013.65 (2013).

34. Milligan, C. \& Gifondorwa, D. Isolation and culture of postnatal spinal motoneurons. Methods in molecular biology 793, 77-85, https://doi.org/10.1007/978-1-61779-328-8_5(2011).

35. Chapnik, E. et al. miR-142 orchestrates a network of actin cytoskeleton regulators during megakaryopoiesis. eLife 3, e01964, https:// doi.org/10.7554/eLife.01964 (2014).

36. Zhou, F. et al. miRNA-9 expression is upregulated in the spinal cord of G93A-SOD1 transgenic mice. International journal of clinical and experimental pathology 6, 1826-1838 (2013)

37. Nolan, K. et al. Increased expression of microRNA-29a in ALS mice: functional analysis of its inhibition. Journal of molecular neuroscience: MN 53, 231-241, https://doi.org/10.1007/s12031-014-0290-y (2014).

38. Issler, O. et al. MicroRNA 135 is essential for chronic stress resiliency, antidepressant efficacy, and intact serotonergic activity. Neuron 83, 344-360, https://doi.org/10.1016/j.neuron.2014.05.042 (2014).

39. Liu, C. M. et al. MicroRNA-138 and SIRT1 form a mutual negative feedback loop to regulate mammalian axon regeneration. Genes \& development 27, 1473-1483, https://doi.org/10.1101/gad.209619.112 (2013).

40. Li, J. et al. miR-30 regulates mitochondrial fission through targeting $\mathrm{p} 53$ and the dynamin-related protein-1 pathway. PLoS genetics 6, e1000795, https://doi.org/10.1371/journal.pgen.1000795 (2010).

41. Lagos-Quintana, M. et al. Identification of tissue-specific microRNAs from mouse. Current biology: CB 12, 735-739 (2002).

42. Parsons, X. H., Parsons, J. F. \& Moore, D. A. Genome-Scale Mapping of MicroRNA Signatures in Human Embryonic Stem Cell Neurogenesis. Molecular Medicine \& Therapeutics 1, https://doi.org/10.4172/2324-8769.1000105 (2012).

43. Bhagat, L., Singh, V. P., Dawra, R. K. \& Saluja, A. K. Sodium arsenite induces heat shock protein 70 expression and protects against secretagogue-induced trypsinogen and NF-kappaB activation. Journal of cellular physiology 215, 37-46, https://doi.org/10.1002/ jcp.21286 (2008).

44. Huang, G. et al. Death receptor 6 (DR6) antagonist antibody is neuroprotective in the mouse SOD1G93A model of amyotrophic lateral sclerosis. Cell death \& disease 4, e841, https://doi.org/10.1038/cddis.2013.378 (2013).

45. van Dongen, S., Abreu-Goodger, C. \& Enright, A. J. Detecting microRNA binding and siRNA off-target effects from expression data. Nature methods 5, 1023-1025, https://doi.org/10.1038/nmeth.1267 (2008).

46. Huang da, W., Sherman, B. T. \& Lempicki, R. A. Bioinformatics enrichment tools: paths toward the comprehensive functional analysis of large gene lists. Nucleic acids research 37, 1-13, https://doi.org/10.1093/nar/gkn923 (2009).

47. Penefsky, H. S. Mechanism of inhibition of mitochondrial adenosine triphosphatase by dicyclohexylcarbodiimide and oligomycin: relationship to ATP synthesis. Proceedings of the National Academy of Sciences of the United States of America 82, 1589-1593 (1985).

48. Agarwal, V., Bell, G. W., Nam, J. W. \& Bartel, D. P. Predicting effective microRNA target sites in mammalian mRNAs. Elife 4, https:// doi.org/10.7554/eLife.05005 (2015). 
49. Makeyev, E. V., Zhang, J., Carrasco, M. A. \& Maniatis, T. The MicroRNA miR-124 promotes neuronal differentiation by triggering brain-specific alternative pre-mRNA splicing. Molecular cell 27, 435-448, https://doi.org/10.1016/j.molcel.2007.07.015 (2007).

50. Clark, P. M. et al. Argonaute CLIP-Seq reveals miRNA targetome diversity across tissue types. Sci Rep 4, 5947, https://doi. org/10.1038/srep05947 (2014).

51. Chi, S. W., Zang, J. B., Mele, A. \& Darnell, R. B. Argonaute HITS-CLIP decodes microRNA-mRNA interaction maps. Nature 460, 479-486, https://doi.org/10.1038/nature08170 (2009).

52. Furuta, M. et al. miR-124 and miR-203 are epigenetically silenced tumor-suppressive microRNAs in hepatocellular carcinoma. Carcinogenesis 31, 766-776, https://doi.org/10.1093/carcin/bgp250 (2010).

53. Zelenko, Z. et al. Silencing vimentin expression decreases pulmonary metastases in a pre-diabetic mouse model of mammary tumor progression. Oncogene 36, 1394-1403, https://doi.org/10.1038/onc.2016.305 (2017).

54. Ho, V. M. et al. GluA2 mRNA distribution and regulation by miR-124 in hippocampal neurons. Mol Cell Neurosci 61, 1-12, https:// doi.org/10.1016/j.men.2014.04.006 (2014).

55. Yoo, A. S., Staahl, B. T., Chen, L. \& Crabtree, G. R. MicroRNA-mediated switching of chromatin-remodelling complexes in neural development. Nature 460, 642-646, https://doi.org/10.1038/nature08139 (2009).

56. Yoo, A. S. et al. MicroRNA-mediated conversion of human fibroblasts to neurons. Nature 476, 228-231, https://doi.org/10.1038/ nature10323 (2011)

57. Morris, R. L. \& Hollenbeck, P. J. The regulation of bidirectional mitochondrial transport is coordinated with axonal outgrowth. J Cell Sci 104(Pt 3), 917-927 (1993).

58. Ruthel, G. \& Hollenbeck, P. J. Response of mitochondrial traffic to axon determination and differential branch growth. The Journal of neuroscience: the official journal of the Society for Neuroscience 23, 8618-8624 (2003).

59. Mattson, M. P., Gleichmann, M. \& Cheng, A. Mitochondria in neuroplasticity and neurological disorders. Neuron 60, 748-766, https://doi.org/10.1016/j.neuron.2008.10.010 (2008).

60. Matveeva, E. A., Venkova, L. S., Chernoivanenko, I. S. \& Minin, A. A. Vimentin is involved in regulation of mitochondrial motility and membrane potential by Rac1. Biol Open 4, 1290-1297, https://doi.org/10.1242/bio.011874 (2015).

61. Marcuzzo, S. et al. Up-regulation of neural and cell cycle-related microRNAs in brain of amyotrophic lateral sclerosis mice at late disease stage. Molecular brain 8, 5, https://doi.org/10.1186/s13041-015-0095-0 (2015).

62. Campos-Melo, D., Droppelmann, C. A., He, Z., Volkening, K. \& Strong, M. J. Altered microRNA expression profile in Amyotrophic Lateral Sclerosis: a role in the regulation of NFL mRNA levels. Molecular brain 6, 26, https://doi.org/10.1186/1756-6606-6-26 (2013).

63. Magrane, J., Sahawneh, M. A., Przedborski, S., Estevez, A. G. \& Manfredi, G. Mitochondrial dynamics and bioenergetic dysfunction is associated with synaptic alterations in mutant SOD1 motor neurons. The Journal of neuroscience: the official journal of the Society for Neuroscience 32, 229-242, https://doi.org/10.1523/JNEUROSCI.1233-11.2012 (2012).

64. De Vos, K. J. et al. Familial amyotrophic lateral sclerosis-linked SOD1 mutants perturb fast axonal transport to reduce axonal mitochondria content. Human molecular genetics 16, 2720-2728, https://doi.org/10.1093/hmg/ddm226 (2007).

65. Tradewell, M. L., Cooper, L. A., Minotti, S. \& Durham, H. D. Calcium dysregulation, mitochondrial pathology and protein aggregation in a culture model of amyotrophic lateral sclerosis: mechanistic relationship and differential sensitivity to intervention. Neurobiology of disease 42, 265-275, https://doi.org/10.1016/j.nbd.2011.01.016 (2011).

66. Yang, Y. et al. EPAC null mutation impairs learning and social interactions via aberrant regulation of miR-124 and Zif268 translation. Neuron 73, 774-788, https://doi.org/10.1016/j.neuron.2012.02.003 (2012).

67. Gluska, S. et al. Rabies Virus Hijacks and accelerates the p75NTR retrograde axonal transport machinery. PLoS Pathog 10, e1004348, https://doi.org/10.1371/journal.ppat.1004348 (2014).

68. Gluska, S., Chein, M., Rotem, N., Ionescu, A. \& Perlson, E. Tracking Quantum-Dot labeled neurotropic factors transport along primary neuronal axons in compartmental microfluidic chambers. Methods Cell Biol 131, 365-387, https://doi.org/10.1016/bs. mcb.2015.06.016 (2016).

69. Ruggiero, A. et al. Loss of forebrain MTCH2 decreases mitochondria motility and calcium handling and impairs hippocampaldependent cognitive functions. Sci Rep 7, 44401, https://doi.org/10.1038/srep44401 (2017).

70. Kim, D. et al. TopHat2: accurate alignment of transcriptomes in the presence of insertions, deletions and gene fusions. Genome biology 14, R36, https://doi.org/10.1186/gb-2013-14-4-r36 (2013).

71. Anders, S., Pyl, P. T. \& Huber, W. HTSeq-a Python framework to work with high-throughput sequencing data. Bioinformatics 31, 166-169, https://doi.org/10.1093/bioinformatics/btu638 (2015).

72. Love, M. I., Huber, W. \& Anders, S. Moderated estimation of fold change and dispersion for RNA-seq data with DESeq. 2. Genome biology 15, 550, https://doi.org/10.1186/PREACCEPT-8897612761307401 (2014).

\section{Acknowledgements}

We thank Jing Liang and Ayelet Avin for technical support, Oded Singer for viral prep, Smadar Levin Zaidman for electron microscopy, Atan Gross, Ido Amit and Eli Arama for reagents and advice (Weizmann Institute of Science); Adi Laser-Azogui and Roy Beck (Tel Aviv University) for generously sharing Vimentin overexpression lentivirus. Heather D. Durham, (McGill University), Michael J. Strong (Western University), Edor Kabashi (ICM - Hôpital Pitié Salpêtrière) for discussions and partnership in ERARE-2 network program of Era-Net ('RNAALS') that supported the work. Additional funding was from grants of Israel Ministry of Health (10928), Israel Science Foundation, Legacy-heritage Fund, Research ERC consolidator program (617351), Target ALS (118945), AFM Telethon (20576), Bruno and Ilse Frick Foundation for Research on ALS, ALS-Therapy Alliance, Yeda CEO fund, Minna-James-Heineman Stiftung through Minerva, Minerva Foundation with funding from the Federal German Ministry for Education and Israel Ministry of Trade and Industry, Nella and Leon Benoziyo Center for Neurological Diseases, the Weizmann Institute of Science, A. Alfred Taubman via IsrALS, Kekst Family Institute for Medical Genetics, David and Fela Shapell Family Center for Genetic Disorders Research, Crown Human Genome Center, Yeda Sela Center, Y. Leon Benoziyo Institute for Molecular Medicine, Helen and Martin Kimmel Institute for Stem Cell Research, the Maurice and Vivienne Wohl Biology Endowment, the Nathan, Shirley, Philip and Charlene Vener New Scientist Fund, Julius and Ray Charlestein Foundation, Celia Benattar Memorial Fund for Juvenile Diabetes, the Wolfson Family Charitable Trust and Adelis Foundation. T.Y. was supported by postdoctoral fellowship from Teva Pharmaceutical Industries Ltd as part of the Israeli National Network of Excellence in Neuroscience (NNE) and by the postdoctoral fellowship from the Sara Lee Schupf foundation. EH is incumbent of Mondry Family Professorial Chair and the Hornstein lab is supported by Dr. Sydney Brenner.

\section{Author Contributions}

T.Y., R.F., and E.H. Designed research and wrote the paper. T.Y., R.F., Y.J., T.G.P., N.K., I.R., E.Y., S.N., H.W.T., M.E.B., T.S., A.P., E.P., E.H. performed research or/and analyzed data. H.M.B., contributed analytic tools. 


\section{Additional Information}

Supplementary information accompanies this paper at https://doi.org/10.1038/s41598-017-17878-x.

Competing Interests: The authors declare that they have no competing interests.

Publisher's note: Springer Nature remains neutral with regard to jurisdictional claims in published maps and institutional affiliations.

(c) (1) Open Access This article is licensed under a Creative Commons Attribution 4.0 International License, which permits use, sharing, adaptation, distribution and reproduction in any medium or format, as long as you give appropriate credit to the original author(s) and the source, provide a link to the Creative Commons license, and indicate if changes were made. The images or other third party material in this article are included in the article's Creative Commons license, unless indicated otherwise in a credit line to the material. If material is not included in the article's Creative Commons license and your intended use is not permitted by statutory regulation or exceeds the permitted use, you will need to obtain permission directly from the copyright holder. To view a copy of this license, visit http://creativecommons.org/licenses/by/4.0/.

(C) The Author(s) 2017 